\title{
REMARKS ON CONVERSE CARLEMAN AND KREIN CRITERIA FOR THE CLASSICAL MOMENT PROBLEM
}

\author{
ANTHONY G. PAKES \\ (Received 5 March 2000; revised 22 November 2000) \\ Communicated by V. Stefanov
}

\begin{abstract}
The key theme is converse forms of criteria for deciding determinateness in the classical moment problem. A method of proof due to Koosis is streamlined and generalized giving a convexity condition under which moments $\mu_{n}=\int_{0}^{\infty} x^{n} f(x) d x$ satisfying $\sum \mu_{n}^{-c / n}<\infty$ implies that $\int_{x^{\prime}}^{\infty} x^{-1-c}(-\log f(x)) d x<\infty$, $c$ a positive constant. A contrapositive version is proved under a rapid variation condition on $f(x)$, generalizing a result of Lin. These results are used to obtain converses of the Stieltjes versions of the Carleman and Krein criteria. Hamburger versions are obtained which relax the symmetry assumption of Koosis and Lin, respectively. A sufficient condition for Stieltjes determinateness of a discrete law is given in terms of its mass function. These criteria are illustrated through several examples.
\end{abstract}

2000 Mathematics subject classification: primary 44A60, 62E10; secondary 26A51, 60E05.

Keywords and phrases: classical moment problem, Carleman and Krein conditions, Fenchel transform, rapid variation.

\section{Introduction}

Let $F(x)$ be a distribution function (DF) on $\mathbb{R}$ with finite moments of all positive orders, $\mu_{n}=\int_{\mathbb{R}} x^{n} d F(x),(n \in \mathbb{N}=\{0,1, \ldots\})$. The classical Hamburger moment problem is to find conditions on the moment sequence $\mathscr{M}=\left\{\mu_{n}\right\}$ ensuring that it is determining, meaning that $F$ is the only DF having this sequence of moments. We then say that $F$ is determined by $\mathscr{M}$, or just that it is determinate, abbreviated as $\mathrm{H}$-det. We say that $F$ is indeterminate (or $\mathrm{H}$-indet) when $\mathscr{M}$ is non-determining, that is, there is another DF having the same moment sequence. We are concerned in this partly expository paper with some aspects of the Carleman and Krein conditions, effectively the only viable criteria which can be used to decide when $F$ is $\mathrm{H}$-det or

(C) 2001 Australian Mathematical Society $0263-6115 / 2001 \$ A 2.00+0.00$ 
$\mathrm{H}$-indet, respectively. In particular we are concerned with recently published converse forms of these criteria.

We say that $F$ is HC-det if its moment sequence satisfies the Carleman condition

$H C(\mathscr{M}): \quad \sigma_{H}(\mathscr{M})=\sum_{n \geq 1} \mu_{2 n}^{-1 / 2 n}=\infty$.

This is a sufficient condition for $F$ to be H-det; see Heyde [5] for a proof that it is not necessary.

Now suppose $f(x)=F^{\prime}(x)$ exists on $\mathbb{R}$. The usual form of Krein's condition, or criterion (Stoyanov [18] for a general review), states that $F$ is H-indet if

$$
I(f)=\int_{-\infty}^{\infty} \frac{-\log f(x)}{1+x^{2}} d x<\infty
$$

This is a strong condition requiring in particular that $f(x)>0$ a.e. in $\mathbb{R}$. Pedersen [12] obtains a substantial weakening of (1) by showing that the region of integration can be replaced by a set $\mathscr{D}$ of positive lower uniform density. This means that $\mathscr{D}$ contains the union over $n \in \mathscr{Z}$ of intervals $\left[a_{n}-\delta_{n}, a_{n}+\delta_{n}\right]$ where the numbers $\delta_{n}$ and $a_{n}-a_{n-1}$ are bounded away from zero and infinity.

Any set of the form $\left(-\infty,-x^{\prime}\right] \cup\left[x^{\prime}, \infty\right)$ with $x^{\prime}>0$ has positive lower uniform density. Hence we will assume that there exists such a set, that $f(x)>0$ a.e. in it, and we say that $F$ is $\mathrm{HK}$-indet if

$H K(f)$ :

$$
I_{H}\left(f ; x^{\prime}\right)=\int_{|x| \geq x^{\prime}} \frac{-\log f(x)}{1+x^{2}} d x<\infty .
$$

This is a sufficient condition for $F$ to be $\mathrm{H}$-indet which has the pleasing feature of imposing a condition only on the tails of $F$. Pedersen [12] gives a still weaker criterion, allowing him to construct a simple explicit example of an $\mathrm{H}$-indet law having $I(f)=\infty$.

These sufficient conditions can be used along with simple comparison arguments to resolve the situation for a very wide range of statistically interesting continuous laws and their powers. Pakes et al. [11] demonstrate this using a unified approach which subsumes all previous specific examples. We are concerned here with certain converse criteria valid under additional growth conditions on the density function $f(\cdot)$.

It is little known among statisticians that Koosis [7] gives conditions on $f(\cdot)$ which ensure that $I(f)<\infty$ holds if $\sigma_{H}(\mathscr{M})<\infty$. Independently of this work, and in the other direction, Lin [8] provides extra conditions on $f(\cdot)$ under which $I(f)=\infty$ implies $H C(\mathscr{M})$. Lin's conditions are tantamount to the assumption that $f($.$) belongs to a sub-family of functions which are rapidly varying at infinity with$ index $-\infty$ (Bingham et al. [2, page 83]). 
The conclusions of these workers are equivalent in the contrapositive sense that Koosis proves that $I(f)=\infty$ plus technical conditions implies $\sigma_{H}(\mathscr{M})=\infty$, but Lin shows that $\sigma_{H}(\mathscr{M})<\infty$ plus technical conditions implies that $I(f)<\infty$. As might be anticipated from this, their key technical assumptions are related-see the remarks in Section 2 following the proof of Corollary 1. However their proofs are quite different. Both authors assume $f(\cdot)$ is symmetric about zero.

Our principal aim is to provide a more unified account of these results and to generalize them by placing no restriction on the form of $f(\cdot)$ inside a bounded interval $\left[-x^{\prime}, x^{\prime}\right]$, and by relaxing the symmetry assumption. We follow historical precedent by starting with the Stieltjes moment problem. Thus in the next section we prove two basic results assuming only that $f(x) \geq 0$ is defined on $\mathbb{R}_{+}$with finite moments $\mu_{n}=\int_{0}^{\infty} x^{n} f(x) d x$.

First, by following the Koosis line of reasoning we show under a weakened form of his convexity condition that if $\sum \mu_{n}^{-c / n}<\infty$ for a positive constant $c$, then $\int_{x^{\prime}}^{\infty} x^{-1-c}(-\log f(x)) d x<\infty$. Our analytically focused proof seems more transparent than Koosis' geometrically flavoured account (which is spread over several sections in two chapters of his book). We achieve this by using some basic results about the Legendre-Fenchel transform. Also, in Step 4 of the proof of Theorem 1, we supply a proof of an inequality asserted by Koosis [7, page 131] with an explanation we are unable to fathom. The proof of Theorem 1 simplifies a lot if the convexity condition ((b) in the assertion) is tightened to strict convexity. Second, we show that Lin's [8] proof of his contrapositive statement is essentially unchanged if $f(\cdot)$ is assumed to be rapidly varying. The resulting criterion can be expressed directly in terms of the index function in the canonical integral representation of $f(\cdot)$.

These results are used in Section 3 to give the Stieltjes versions of the converse Carleman and Krein conditions. In addition we give some examples, showing in particular that Lin's assertion of his converse Krein condition is not quite correct. This section ends with some reflections on decidability for discrete laws. Pedersen [12, Corollary 3.4] gives a Krein condition for a discrete law to be H-indet. In Proposition 2 we show this induces a comparable result for the Stieltjes problem. In addition we give in Theorem 6 a discrete version of Theorem 2, and this specialises to conditions under which a discrete law is S-det.

We return to the Hamburger problem in Section 4, using the basic theorems to give our generalizations of the Koosis and Lin theorems. It seems not possible to entirely drop the symmetry assumption, but we can replace it with a balance condition on the extreme tails of $F$. This is done either by imposing a condition on the structural form of the even-order moments, or on $f(\cdot)$. Section 4 begins with a short review of known results about converse formulations of the classical criteria.

In Section 5 we consider the effect of logarithmic and exponential transformations on decidability criteria for determinateness. Roughly speaking, if $Y>0$ then the 
distribution of $\log Y$ can be $\mathrm{H}$-indet only if $P(Y>y)$ and $P\left(Y<y^{-1}\right)$ decrease like a slowly varying function. The exponential transformation gives 'logged' distributions such as the log-normal and log-gamma. We end the paper by finding those values of $r$ for which the distribution of $X^{r}$ is S-det, where $X=e^{-S(\alpha)}$ and $S(\alpha)$ has a spectrally positive stable law.

\section{Basic results}

The proof of our first basic result follows Koosis' (1988) arguments, but it is rendered more transperant by using basic properties of the Legendre-Fenchel transform (Hiriart-Urruty and Lemaréchal [6, Chapter 1]). The argument used in Step 4 seems far more transperant than the proof of the second lemma of Section IV D in Koosis [7], which he invokes on page 131.

THEOREM 1. Suppose that $f(\cdot)$ is defined and non-negative on $\mathbb{R}_{+}$and that it satisfies the following conditions.

(a) $\mu_{n}=\int_{0}^{\infty} x^{n} f(x) d x$ is finite for all $n$.

(b) There exists $x^{\prime} \geq 0$ such that $0<f(x)<\infty$ for $x>x^{\prime}$ and

$$
\psi(y)=-\log f\left(e^{y}\right)
$$

is convex in $\left(y^{\prime}, \infty\right)$, where $y^{\prime}=\log x^{\prime}$.

(c) For some $c>0$,

$$
\sigma(\mathscr{M} ; c)=\sum_{n \geq 0} \mu_{n}^{-c / n}<\infty
$$

Then

$$
K\left(f ; c, x^{\prime}\right)=\int_{x^{\prime}}^{\infty} x^{-1-c}(-\log f(x)) d x<\infty
$$

PROOF. A change of variables shows that

$$
K\left(f ; c, x^{\prime}\right)=J\left(\psi ; c, y^{\prime}\right)=\int_{y^{\prime}}^{\infty} e^{-c y} \psi(y) d y .
$$

The thrust of the proof is to show that the hypotheses imply $J\left(\psi ; c, y^{\prime}\right)<\infty$. The proof proceeds in several steps.

(1) Since $\int_{0}^{1} x^{n} f(x) d x \rightarrow 0$, we have

$$
\mu_{n}=o(1)+\int_{x>1} x^{n}\left(x^{2} f(x)\right) \frac{d x}{x^{2}}
$$


A little thought shows that condition (b) implies that $\psi(y)$ increases convexly to $\infty$ as $y \uparrow \infty$. Hence $f(x) \downarrow 0$ as $x \uparrow \infty$. In addition, it follows from (a) that $f(x)$ decreases to zero faster than any power $x^{-A}, A \gg 0$. Consequently,

$$
M_{n}=\sup _{x \geq x^{\prime}}\left[x^{n+2} f(x)\right] \quad(n \in \mathbb{N})
$$

is finite and the supremum is achieved in a compact interval, since $f(\cdot)$ is continuous. Clearly then, $\mu_{n} \leq M_{n}+o(1)$, and hence (c) implies that

$$
\sum M_{n}^{-c / n}<\infty
$$

(2) Observe that

$$
\log M_{n}=l_{n} \equiv \sup _{y \geq y^{\prime}}[n y-g(y)] \quad(n \in \mathbb{N}),
$$

where $g(y)=\psi(y)-2 \log y$ is convex. The sequence $\left\{l_{n}\right\}$ is convex and increasing. This follows from standard results about the Legendre-Fenchel transform $\gamma(z)=$ $\sup _{y \geq y^{\prime}}[z y-g(y)](z \geq 0)$ which is convex and non-decreasing in $\mathbb{R}_{+}$(HiriartUrruty and Lemaréchal [6, page 36]). It follows that the sequence $\left\{M_{n} / M_{n+1}\right\}$ is non-increasing whence

$$
\frac{M_{1}}{M_{n+1}}=\frac{M_{1}}{M_{2}} \frac{M_{2}}{M_{3}} \cdots \frac{M_{n}}{M_{n+1}}>\left(\frac{M_{n}}{M_{n+1}}\right)^{n},
$$

and we conclude from (2) that

$$
\sum_{n \geq 1}\left(M_{n} / M_{n+1}\right)^{c}<\infty .
$$

(3) Along the lines of Koosis [7, page 130] define the function

$$
\phi(y)=\sup _{n \in \mathbb{N}}\left[y n-l_{n}\right], \quad\left(y \geq y^{\prime}\right) .
$$

We show in this step that (3) implies

$$
J\left(\phi ; c, y^{\prime}\right)<\infty .
$$

A consideration of the convex piecewise-linear function which interpolates the points $\left(n, l_{n}\right)$ shows that $\phi(y)$ is a piecewise-linear and convex function. Let $\phi_{n}(y)=y n-l_{n}$ $(n \in \mathbb{N})$ and $\Delta \phi_{n}(y)=\phi_{n}(y)-\phi_{n-1}(y)=y-p_{n}$, where $p_{n}=l_{n}-l_{n-1}$. Since $\left\{l_{n}\right\}$ is convex, $\left\{p_{n}\right\}$ is a non-decreasing sequence. Since $l_{n} \rightarrow \infty$, the $p_{n}$ are ultimately all positive, and hence there is no loss of generality in supposing they are positive for 
all $n$. In addition we may assume that they are distinct since equal pairs make a zero contribution in what follows.

If $p_{n}<y<p_{n+1}$ then $\Delta \phi_{\nu}(y)$ changes sign from positive to negative as $v$ jumps from $n$ to $n+1$. Hence

$$
\phi(y)=y n-l_{n} \quad \text { and } \quad \phi^{\prime}(y)=n \quad\left(p_{n} \leq y \leq p_{n+1}\right) .
$$

Observe that

$$
J\left(\phi ; c, y^{\prime}\right)=\sum_{n \geq 0} \int_{p_{n}}^{p_{n+1}} e^{-c y} \phi(y) d y .
$$

Integration by parts gives

$$
\int_{p_{n}}^{p_{n+1}} e^{-c y} \phi(y) d y=\phi\left(p_{n}\right) e^{-c p_{n}}-\phi\left(p_{n+1}\right) e^{-c p_{n+1}}+\int_{p_{n}}^{p_{n+1}} e^{-c y} \phi^{\prime}(y) d y .
$$

Summing yields

$$
\begin{aligned}
J\left(\phi ; c, y^{\prime}\right) & =\lim _{N \rightarrow \infty} \sum_{n=0}^{N}\left[\phi\left(p_{n}\right) e^{-c p_{n}}-\phi\left(p_{n+1}\right) e^{-c p_{n+1}}+n\left(e^{-c p_{n}}-e^{-c p_{n+1}}\right)\right] \\
& \leq \phi\left(p_{0}\right) e^{-c p_{0}}+\sum_{n=1}^{\infty} n\left(\left(\frac{M_{n-1}}{M_{n}}\right)^{c}-\left(\frac{M_{n}}{M_{n+1}}\right)^{c}\right) .
\end{aligned}
$$

The summands are non-negative, whence expressing $n$ as a sum of units and reversing the order of the resulting double sum yields the series (3). This proves (4).

(4) The final step is to show that (4) implies $J\left(\psi ; c, y^{\prime}\right)<\infty$. The principal step is showing that (4) implies

$$
J\left(g ; c, y^{\prime}\right)<\infty .
$$

Observe in passing that the supremum defining $\phi(y)$ is attained at some $v \in \mathbb{N}$ (depending on $y$ ), that is, $\phi(y)=y v-l_{v}$. The definition of $l_{n}$ implies that $l_{n} \geq$ $n y-g(y)\left(y \geq y^{\prime}\right)$, and hence that $\phi(y) \leq g(y)$. This shows that (5) implies (4). We obtain the desired converse inequality by showing that

$$
g(y) \leq \phi(y)+y
$$

for sufficiently large $y$.

To see this observe that the Legendre-Fenchel transform $\gamma(z)$ is defined for all real $z$, where it still is convex and non-decreasing. The Fenchel duality theorem asserts the inverse relation

$$
g(y)=\sup _{z \in \mathbb{R}}[y z-\gamma(z)], \quad\left(y \geq y^{\prime}\right) .
$$


To understand the structure of $\gamma(z)$ on the whole line, it suffices to consider the case where $g(\cdot)$ has a continuous derivative in $\left(y^{\prime}, \infty\right)$. For this it suffices that $g^{\prime}(y)$ exists everywhere in $\left(y^{\prime}, \infty\right)$. Choose $A \ll 0$ and let $\omega(x)=x^{A} f(x)$. Then $g(y) / y=$ $A-y^{-1} \log \omega\left(e^{y}\right)$. Since $\omega(x) \rightarrow 0(x \rightarrow \infty)$, we see that $\liminf _{y \rightarrow x} g(y) / y \geq A$, that is, $g(y) / y \rightarrow \infty$. Hence $g(y)>y$ for all $y \geq y^{\prime \prime}$, say, and since $g(\cdot)$ is convex we have that

$$
g(y) / y \leq g^{\prime}(y) \quad\left(y \geq y^{\prime \prime}\right) \quad \text { and } \quad g^{\prime}(y) \uparrow \infty .
$$

Consequently we can choose $y^{\prime}$ large enough to ensure that $z^{\prime}=g^{\prime}\left(y^{\prime}\right)>0$, and that $g(y)>0$ in $\left[y^{\prime}, \infty\right)$. It follows that the objective function defining $\gamma(\cdot)$ has derivative $z-g^{\prime}(y)<z-z^{\prime} \leq 0$ if $z \leq z^{\prime}$. Hence for such $z$ the supremum is attained at $y=y^{\prime}$, and then $\gamma(z)=z y^{\prime}-g\left(y^{\prime}\right)$. It follows that for $z \leq z^{\prime}$ the objective function in (6) equals $z\left(y-y^{\prime}\right)+g\left(y^{\prime}\right)$, and since this is increasing in $z$ when $y>y^{\prime}$, we conclude that the supremum in (6) is achieved in $\left[z^{\prime}, \infty\right)$, and hence in the larger set $[0, \infty)$.

Using this, and the fact that $l_{n}=\gamma(n)$, we have

$$
\begin{aligned}
g(y) & =\sup _{z \geq 0}[y z-\gamma(z)]=\sup _{n \in \mathbb{N}} \max _{n \leq z \leq n+1}[y z-\gamma(z)] \\
& \leq \sup _{n \in \mathbb{N}}[y(n+1)-\gamma(n)]=\phi(y)+y .
\end{aligned}
$$

This establishes the equivalence of (4) and (5) under the convexity condition (b). But since $\psi(y)=g(y)+2 \log y$, we conclude that (5) is equivalent to $J\left(\psi ; c, y^{\prime}\right)<\infty$.

REMARK. The details of the proof do not require $F(x)$ to be differentiable in $\left(0, x^{\prime}\right)$. Since the convexity assumption (b) implies $g^{\prime}(y)$ exists a.e. in $\left(y^{\prime}, \infty\right)$, the argument in Step (4) entails no loss of generality.

Much of what is in Step 3 and Step 4 can be avoided as follows, at least under a slightly stronger smoothness assumption. Since the secant function $\gamma(z) / z$ is eventually non-decreasing it follows from integral test comparisons that (2) is equivalent to

$$
\int_{z}^{\infty} e^{-\gamma(z) / z} d z<\infty
$$

Choose $B \gg 0$ and $y(B)$ such that $g(y) \leq B$ if $y^{\prime} \leq y \leq y(B)$. Then

$$
\gamma(z) / z \geq \sup _{y \leq \leq \leq y(B)}[y-g(y) / z] \geq \sup _{y, \leq y \leq y(B)}[y-B / z]=y(B)-B / z,
$$

whence $\liminf :-\alpha \gamma(z) / z \geq y(B)$. Clearly $y(B) \rightarrow \infty$ as $B \rightarrow \infty$, showing that $\gamma(z) / z \rightarrow \infty$ as $z \rightarrow \infty$. The derivative $\gamma^{\prime}(z)$ exists provided $g(\cdot)$ is strictly convex 
(Hiriart-Urruty and Lemaréchal [6, page 40]) and then $\gamma(z) / z \leq \gamma^{\prime}(z)$ if $z$ is large enough. We conclude that (2) implies that

$$
\tilde{J}=\int_{z^{\prime}}^{\infty} e^{-c \gamma^{\prime}(z)} d z<\infty .
$$

Indeed, this is generally valid if the derivative is replaced by either the right-hand or left-hand derivative functions of $\gamma(\cdot)$. Anyway the generalized inverse of $\gamma^{\prime}(z)$ coincides with the left-hand derivative function $g_{-}^{\prime}(y)$ of $g(\cdot)$ (Hiriart-Urruty and Lemaréchal [6, page 39]). This derivative function is non-decreasing and leftcontinuous. Substituting $y=\gamma^{\prime}(z)$ in the above integral yields

$$
\tilde{J}=\int_{y^{\prime}}^{\infty} e^{-c y} d g_{-}^{\prime}(y) .
$$

Writing this infinite Stieltjes integral as the limit as $y^{\prime \prime} \rightarrow \infty$ of an integral over $\left[y^{\prime}, y^{\prime \prime}\right]$, a double integration by parts yields

$$
\tilde{J} \geq \int_{y^{\prime}}^{\infty} e^{-y} g(y) d y,
$$

and the assertion of the theorem follows.

The following corollary is an apparent generalization of Theorem 1. Koosis' proof is for the case $c=1 / 2$ and $l=2$.

COROLlaRY 1. Suppose Conditions (a) and (b) are satisfied and that (c) is replaced with

$\left(\mathrm{c}^{\prime}\right)$ For some $c>0$ and $l \in \mathbb{N}, \sum_{n \geq 0} \mu_{l n}^{-c / n}<\infty$.

Then

$$
K\left(f ; c, l, x^{\prime}\right)=\int_{x^{\prime}}^{\infty} x^{-1-c}\left(-\log f\left(x^{1 / l}\right)\right) d x<\infty,
$$

or equivalently, $K\left(f ; l c, x^{\prime}\right)<\infty$.

PROOF. A change of variables shows that $\left\{\mu_{l n}\right\}$ is the moment sequence of the density function $f_{l}(x)=l^{-1} x^{-1+1 / l} f\left(x^{1 / l}\right)$. The corresponding $g(\cdot)$-function is

$$
g_{l}(y)=\log l-(1-1 / l) y+g(y / l)
$$

which satisfies Condition (b) of Theorem 1 . Hence $\left(\mathrm{c}^{\prime}\right)$ implies $K\left(f_{i} ; c, x^{\prime}\right)<\infty$, and the assertion follows after a little algebra. 
Lin [8] assumes $f^{\prime}(x)$ exists and is positive in $(0, \infty)$ and that $\zeta(x)=-x f^{\prime}(x) / f(x)$ is eventually increasing to $\infty$. An integration yields the explicit form $f(x)=$ $f(1) \exp \left[-\int_{1}^{x} v^{-1} \zeta(v) d v\right]$. This is a special case of the representation of the Karamata class $K R_{-\infty}$ of rapidly varying functions with index $-\infty$. See Bingham et al. [2] for the reciprocal class $K R_{\infty}$.

At this point we observe that if $f^{\prime}(x)$ exists in $(0, \infty)$ then Koosis [7] convexity condition, that $\psi(y)$ is convex on the real line, is equivalent to the condition that $\psi^{\prime}(y)$ is non-decreasing, that is, that $\zeta(x)$ is non-decreasing in $(0, \infty)$. This is the link between Lin's and Koosis' assumptions mentioned in Section 1.

For our generalization of Lin's condition we admit a larger subset of $K R_{-\infty}$ by assuming that

$$
\text { (A) } f(x)=A(x) e^{-R(x)} \equiv A(x) \exp \left[-\eta(x)-\int_{x^{\prime}}^{x} v^{-1} \zeta(v) d v\right] \text {, }
$$

where $0 \leq A(x) \rightarrow A \in(0, \infty)$;

(B) $\quad \eta(x)$ is non-decreasing for $x \geq x^{\prime}$; and

(C) $\zeta\left(x^{\prime}\right)>0, \zeta(x) \rightarrow \infty$ as $x \uparrow \infty$, and there exists $N \in \mathbb{N}$ and numbers $x_{n}$ $(n \geq N)$ such that $n \leq \zeta(x) \leq n+1$ if and only if $x_{n} \leq x \leq x_{n+1}$, and $x_{n} \uparrow \infty$.

Note that we do not require the index function $\zeta(\cdot)$ to be monotone, although this condition is likely to be satisfied and it ensures the $x_{n}$ exist. However the connection with convexity is severed by allowing $\eta(\cdot)$ to be non-convex and by allowing $\zeta(\cdot)$ to oscillate to a limited degree. Thus Theorem 1 and Theorem 2 below have overlapping but different domains of validity. Assumptions (A)-(C) are about the lightest allowable for the proof which follows.

We want the condition

(D) $K\left(f ; c, x^{\prime}\right)=\infty$

to be determined by only the index function $\zeta(\cdot)$ and hence we will further assume

(E) $\int_{x^{\prime}}^{\infty} x^{-1-c} \eta(x) d x<\infty$.

It follows from (E) that (D) is equivalent to

(F) $\int_{x^{\prime}}^{\infty} x^{-1-c} \zeta(x) d x=\infty$.

THEOREM 2. Suppose $f(\cdot)$ is given by (A) and that it satisfies (a), (B), (C) and (E) for some $c>0$. Then $(\mathrm{D})$, equivalently $(\mathrm{F})$, implies that $\sigma(\mathscr{M} ; c)=\infty$.

PROOF. The proof follows Lin's [8] proof in broad outline, which in turn has an antecedent [8, page 87]. By increasing $x^{\prime}$ if necessary, we can assume $R(\cdot)$ is strictly increasing and that $A(x)<2 A$ in $\left[x^{\prime}, \infty\right)$. It follows that for $x \geq x^{\prime}$,

$$
0<f(x) \leq \sup _{x \geq x^{\prime}} f(x)<\infty .
$$

Without loss of generality we can assume the $x_{n}$ 's are distinct because coincident pairs will make no contribution to what follows. Also, we can take $x^{\prime}=x_{N}$. 
In the notation of (A), define $\bar{f}(x)=A e^{-R(x)}$, not necessarily a density function, and $\phi_{n}(x)=x^{n} \bar{f}(x)(n \geq N)$. We have for $x \geq x_{n}$ and $h>0$ that

$$
-\log \frac{\phi_{n}(x+h)}{\phi_{n}(x)}=\eta(x+h)-\eta(x)+\int_{x}^{x+h} \zeta(v) d v / v-n \int_{x}^{x+h} d v / v \geq 0,
$$

since the last term is $-\log \left[((x+h) / x)^{n}\right]$, and we have used (B) and (C). Hence $\phi_{n}(x)$ is non-increasing in $\left[x_{n}, \infty\right)$ and in particular $\phi_{n}(x) \leq \phi_{n}\left(x_{n}\right)$.

The next step follows Lin's upper bounding argument:

$$
\begin{aligned}
\mu_{n} & =\int_{0}^{\infty} x^{n} f(x) d x \leq \int_{0}^{x_{n+2}} x^{n} f(x) d x+2 \int_{x_{n+2}}^{\infty} \phi_{n+2}(x) d x / x^{2} \\
& \leq x_{n+2}^{n}+2 x_{n+2}^{n+2} \bar{f}\left(x_{n+2}\right) \int_{x_{n+2}}^{\infty} x^{-2} d x=x_{n+2}^{n}\left[1+2 x_{n+2} \bar{f}\left(x_{n+2}\right)\right] \leq \text { const } \cdot x_{n+2}^{n},
\end{aligned}
$$

since $x f(x) \rightarrow 0$. Hence $\mu_{n}^{-c / n} \geq$ const $\cdot x_{n+2}^{-c}$ and the conclusion follows if $S(c)=$ $\sum_{n>N} x_{n}^{-c}=\infty$. But, from (C) and (F),

$$
\begin{aligned}
\infty & =\int_{x_{N}}^{\infty} x^{-1-c} \zeta(x) d x=\sum_{n>N} \int_{x_{n-1}}^{x_{n}} x^{-1-c} \zeta(x) d x \\
& \leq \sum_{n>N} n \int_{x_{n-1}}^{x_{n}} x^{-1-c} d x=c^{-1} \sum_{n>N} n\left[x_{n-1}^{-c}-x_{n}^{-c}\right] .
\end{aligned}
$$

This series is essentially the same as the one in Step 3 of the proof of Theorem 1 . Thus it differs by $O(1)$ from $S(c)$.

Corollary 1 has the following analogue.

CoROllary 2. Suppose $l \in \mathbb{N}$, that Conditions (a), (A)-(C) hold, and that $\int_{x^{\prime}}^{\infty} x^{-1-c l} \eta(x) d x<\infty$. If

then

$$
\int_{x^{\prime}}^{\infty} x^{-1-c l} \zeta(x) d x=\infty
$$

$$
\sum \mu_{l n}^{-c / n}=\infty
$$

\section{The Stieltjes moment problem}

The Stieltjes moment problem is the analogue of the Hamburger problem for distributions supported in $\mathbb{R}_{+}$, that is, whose DF's satisfy $F\left(0_{-}\right)=0$. We use the terms $\mathrm{S}$-det and $\mathrm{S}$-indet to denote that $F$ is determinate or indeterminate, respectively, 
for the Stieltjes problem. It is possible for $F$ to be S-det but $\mathrm{H}$-indet, and then it must be a discrete DF. If $F$ is S-det and $F(0)=0$ then it is H-det too. See Heyde [5, Theorem A].

It is well known that the map $x \mapsto x^{2}$ allows the Stieltjes moment problem to be imbedded in the Hamburger problem. See Heyde [5, page 92] and Slud [15, page 2201] on this correspondence. In statistical terms, if a random variable $X \geq 0$ has DF $F$ and moment sequence $\left\{\mu_{n}\right\}$ then, with $B$ a random variable which is independent of $X$ and $P(B=1)=P(B=-1)=1 / 2$, the random variable $Y=B \sqrt{X}$ has a symmetric DF and $E\left(Y^{2 n}\right)=\mu_{n}$. Hence, as is well known, we can say that $F$ is SC-det if

$$
S C(\mathscr{M}): \quad \sum \mu_{n}^{-1 / 2 n}=\infty .
$$

This is a sufficient condition for $F$ to be $\mathrm{S}$-det.

In the other direction, we consider the following Stieltjes version of $H K(f)$ : There exists $x^{\prime}>0$ such that

$S K(f)$ :

$$
I_{S}\left(f ; x^{\prime}\right)=\int_{x^{\prime}}^{\infty} \frac{-\log f\left(x^{2}\right)}{1+x^{2}} d x<\infty,
$$

that is,

$$
\int_{x^{\prime}}^{\infty} x^{-3 / 2}(-\log f(x)) d x<\infty .
$$

We formally state and prove the following relaxed version of Slud [15, Corollary 1].

Proposition 1. If there exists $x^{\prime}>0$ such that $F$ has a density $f(x)$ in $\left(x^{\prime}, \infty\right)$ which satisfies $S K(f)$, then $F$ is $S$-indet.

PROOF. The random variable $Y$ constructed above has a symmetric density $f_{Y}(y)=$ $|y| f\left(y^{2}\right)\left(|y|>y^{\prime}=\sqrt{x^{\prime}}\right)$ which satisfies $H K\left(f_{Y}\right)$. Hence there is a random variable $Z$ which has a DF $G \not \equiv F_{Y}$ but which has the same moment sequence as $F_{Y}$. Consequently $E\left(Z^{2 n}\right)=\mu_{n}$, that is, $Z^{2} \geq 0$ has the moment sequence $\left\{\mu_{n}\right\}$ and a DF which differs from $F$.

A law or DF which is S-indet by virtue of $S K(f)$ is said to be SK-indet. It is clear now that Theorem 1 and Theorem 2 with $c=1 / 2$ give the following converse forms of the Stieltjes versions of the Carleman and Krein criteria.

THEOREM 3 (Converse Carleman Criterion). Suppose $F$ is a DF supported in $\mathbb{R}_{+}$ and having a density function which satisfies Conditions (a) and (b) of Theorem 1. Then $F$ is $S K$-indet if $\sigma_{S}(\mathscr{M})=\sum \mu_{n}^{-1 / 2 n}<\infty$.

THEOREM 4 (Converse Krein Criterion). Suppose $F$ is a DF supported in $\mathbb{R}_{+}$and having a density function which satisfies Conditions (a), (A)-(C), and also that 
$\int_{x^{\prime}}^{\infty} x^{-3 / 2} \eta(x) d x<\infty$. Then $F$ is $S C$-det if $\int_{x^{\prime}}^{\infty} x^{-3 / 2} R(x) d x=\infty$, or equivalently, if $\int_{x^{\prime}}^{\infty} x^{-3 / 2} \zeta(x) d x=\infty$.

EXAMPLE 1. Condition (b) is satisfied by the general log-normal distribution since $\psi(y)$ is then a convex quadratic function. Since the moment sequence has the generic form $\gamma^{n^{2}} \xi^{n}, \gamma>1$ and $\xi \in \mathbb{R}$, we see that $\sigma_{S}(\mathscr{M})<\infty$ and hence any log-normal law is SK-indet.

More generally, suppose $f(x)=\exp \left(-x^{\Delta} L(x)\right)(x>0)$, where $\Delta \geq 0$ and $L(\cdot)$ is a normalized slowly varying function. Condition (b) is satisfied if $\Delta>0$, and also if $\Delta=0$ and the index function in the representation of $L(\cdot)$ is itself a differentiable normalized slowly varying function. The Carleman condition is both necessary and sufficient for $F$ to be S-det. See Pakes et al. [11] for a more detailed discussion of this case.

EXAMPLE 2. Pakes et al. [11] consider a density of the form

$$
f(x ; Q, \lambda)= \begin{cases}K_{Q}^{-1} Q(x) \exp \left(-c x^{1 / \lambda}\right) & \text { if } x>0 \\ 0 & \text { if } x<0\end{cases}
$$

where $Q(x)<\max \left(x^{\delta-1}, x^{k}\right)$ for constants $k \geq 1$ and $0<\delta \leq 1$. This growth constraint was selected to ensure that $K_{Q}$ can be chosen to make the right-hand side a density function. Clearly $S K(f)$ is satisfied if and only if $\int_{x^{\prime}}^{\infty} x^{-3 / 2+1 / \lambda} d x<\infty$, that is, $\lambda>2$.

Theorem 4 cannot be applied without further restricting the form of $Q(\cdot)$. We suppose that $Q(x)=x^{\gamma},(\gamma>0)$ for $x \geq x^{\prime}$ at least. Then (A) is satisfied with $R(x)=c x^{1 / \lambda}-\gamma \log x$, giving $\zeta(x)=x R^{\prime}(x)=(c / \lambda) x^{1 / \lambda}-\gamma$. This satisfies (C) if $x^{\prime}$ is sufficiently large. Consequently (F) is satisfied if and only if $\lambda \leq 2$, and then $F$ is S-det. Corollary 2 in Pakes et al. [11] shows this conclusion extends to all densities of the form (7).

Now let $F$ be an arbitrary DF on $\mathbb{B}_{+}$with moment sequence $\mathscr{M}$. For each $r \geq 0$ we induce a DF $F_{r}$ with moment sequence $\mathscr{H}_{L B}(r)=\left\{\mu_{r}(n)\right\}$ by length biasing of order $r$ (Pakes [10]) via

$$
F_{r}(x)=\mu_{r}^{-1} \int_{0}^{x} v^{r} d F(v) \quad \text { and } \quad \mu_{n}(r)=\mu_{r+n} / \mu_{r} \quad(n \in \mathbb{N}) .
$$

Obviously $\mathscr{M}$ is S-determining if and only if $\mathscr{M}_{L B}(r)$ is. In addition, if the density $f(x)$ exists, then it satisfies Condition (b) if and only if $f_{r}(x)=x^{r} f(x) / \mu_{r}$ satisfies the corresponding condition. Similarly $f(x) \in K R_{-\infty}$ if and only if $f_{r}(x) \in K R_{-\infty}$. Thus applying Theorem 3 or Theorem 4 to $f(x)$ is equivalent to applying them to $f_{r}(x)$. 
Next, let $\bar{F}(x)=1-F(x)$ and define a density function $g_{r}(x)=r x^{r-1} \bar{F}(x) / \mu_{r}$ $(r>0)$. We can define $g_{0}(x)=f(x)$, if it exists. The corresponding moment sequence $\mathscr{M}_{S E}(r)=\left\{\bar{\mu}_{n}(r)\right\}$ is given by

$$
\bar{\mu}_{n}(r)=\frac{r}{r+n} \cdot \frac{\mu_{r+n}}{\mu_{r}}=\frac{r}{r+n} \mu_{n}(r) \quad(n \in \mathbb{N}) .
$$

The operation giving this law and moment sequence is called the stationary-excess operation of order $r$. See Pakes [9, Section 1 and Section 4], but beware the different nomenclature.

Let $\beta(r)$ denote a random variable having the beta $(r, 1)$ law. Clearly $E\left[(\beta(r))^{n}\right]=$ $r /(r+n)$. If $X$ is a random variable with the DF $F$, then $\hat{X}_{r}$ and $\tilde{X}_{r}$ denote its length-biased and stationary-excess version, respectively, both of order $r$. We see that

$$
\tilde{X}_{r} \stackrel{d}{=} \beta(r) \hat{X}_{r}
$$

where the factors on the right-hand side are independent. Hence there is a bijection between the sets of DF's $\mathscr{F}, \mathscr{F}_{L B}(r)$ and $\mathscr{F}_{S E}(r)$ which have, respectively, the moment sequences $\mathscr{M}, \mathscr{M}_{L B}(r)$ and $\mathscr{M}_{S E}(r)$. In addition, the set $\mathscr{F}_{S E}(r)$ is obtained from $\mathscr{F}_{S E}(1)$ by length biasing of order $r-1$.

In particular $\mathscr{M}$ is S-determining if and only if $\mathscr{M}_{S E}(1)$ is, giving the following result.

THEOREM 5. The distribution function $F$ is $S$-indet if $\int_{x^{\prime}}^{\infty} x^{-3 / 2}(-\log \bar{F}(x)) d x<\infty$. Conversely, if $\bar{F} \in K R_{-\infty}$ then $F$ is $S$-det provided $\int_{x^{\prime}}^{\infty} x^{-3 / 2}(-\log \bar{F}(x)) d x=\infty$ and the other conditions of Theorem 2 are satisfied.

We now make good our claim that Lin's converse Krein criterion for the Stieltjes problem is not quite correct. He asserts that if $f(0)=0$ and $f(x)>0$ when $x>0$, then $F$ is $S$-det provided $I_{S}(f ; 0)=\infty$ and his regularity condition is satisfied. The problem is allowing, even insisting, that $f(0)=0$.

EXAMPLE 3. Let $f(x)$ be given by (7) with

$$
Q(x)= \begin{cases}\exp \left(-A x^{-1 / \alpha}\right) & \text { if } x>0 \\ 0 & \text { if } x=0\end{cases}
$$

where $\alpha, A>0$. Then, as we have shown, $F$ is $\mathrm{S}$-indet if and only if $\lambda>2$. But in this case $I_{S}(f ; 0)=\infty$ if and only if $\alpha \leq 2$, contradicting Theorem 4 in Lin [8]. His assertion is correct if $f(0)>0$.

If $f(\cdot)$ is zero in a bounded interval then obviously $I_{S}(f ; 0)=\infty$ and the usual form of the Krein criterion says nothing about the determinacy of $F$. Example 2 
shows this too is the case if $f(\cdot)$ is positive everywhere except at the origin near which its graph is very 'flat'. Similar flat regions occur around $x$ " $>0$ if $Q(\cdot)$ contains a factor of the form $\exp \left(-A\left|x-x^{\prime \prime}\right|^{-1 / \alpha}\right)$ and $\alpha \leq 2$. This underscores the utility of Pedersen's extended version of the Krein criterion.

Pedersen [12] proves a Krein criterion for the $\mathrm{H}$-indeterminacy of a discrete distribution. By modifying details of the proof of Proposition 1 we obtain the following Krein condition for lattice laws supported in $[0, \infty)$. By measuring in units of the lattice spacing we can, and shall, take this to be unity. Hence we assume $p_{j}=P(X=j)>0$ $\left(j \geq j^{\prime}>0\right)$, and $\mu_{n}=E\left(X^{n}\right)<\infty$.

PROPOSITION 2. If

$$
S\left(F ; j^{\prime}\right)=\sum_{j \geq j^{\prime}} \frac{-\log p_{j^{2}}}{1+j^{2}}<\infty
$$

then $F$ is $S$-indet.

Proof. Express $F$ as the mixture $c F_{1}+(1-c) F_{2}$ where $c=\sum_{j \geq 0} p_{j^{2}}$ and $F_{1}$ allocates mass $q_{j}=p_{j^{2}} / c$ to $j^{2}$. Denote by $G$ the symmetric two-sided DF which places mass $q_{j} / 2$ at $\pm j$. The condition (8) implies that $\sum\left(-\log q_{j}\right) /\left(1+j^{2}\right)<\infty$ and we conclude from Corollary 3.4 of Pedersen [12] that $G$ is H-indet. Let $G_{1}$ be a different two-sided DF having the same moment sequence as $G$, and let $\tilde{G}_{1}$ denote the DF on $[0, \infty)$ obtained from $G_{1}$ under the map $x \mapsto x^{2}$. Then, reasoning as in the proof of Proposition 1, $\tilde{G}_{1}$ has the same moment sequence as $F_{1}$, and hence $c \tilde{G}_{1}+(1-c) F_{2} \not \equiv F$ has the moment sequence $\left\{\mu_{n}\right\}$.

Proposition 2 can be used with $S C(\mathscr{M})$ to classify any discrete analogue of the various density families treated by Pakes et al. [11]. For example, if $p_{j} \propto \exp (-L(j))$, where $L(\cdot)$ is slowly varying, then $F$ is $S$-indet. Again, if $p_{j}=R(j) \exp \left(-j^{1 / \lambda}\right)$, where $R(\cdot)$ is regularly varying, then $F$ is $S$-indet if and only if $\lambda>2$.

The kernel of the above proof produces a criterion for non-lattice laws under certain restrictions. Suppose $F$ has an unbounded and countable support $\Lambda$, and $p_{l}=P(X=l)>0(l \in \Lambda)$. If $\Lambda$ is closed under squaring, $\Lambda^{2} \subset \Lambda$, then (8) can be generalized to $\sum_{l \in \Lambda}\left(-\log p_{l^{2}}\right) /\left(1+l^{2}\right)<\infty$. In this case $F$ is $S$-indet provided there is a constant $h>0$ such that $\Lambda$ is relatively $h$-dense, meaning that outside of a bounded set any interval of length $h$ contains at least one element of $\Lambda$. This condition limits the maximum size of gaps in $\Lambda$. For example, lacunary supports are not admitted, although they are often used to construct indeterminate discrete laws. See, for example, Pakes [9] and Stoyanov [17, Section 11.7 and Section 11.8].

This restriction on $\Lambda$ can be relaxed to a small extent by applying the mapping argument directly to $F$. This shows that $F$ is S-indet if $\sum_{l \in \Lambda}\left(-\log p_{l}\right) /(1+l)<\infty$ and $\{\sqrt{l}: l \in \Lambda\}$ is relatively $h$-dense. 
The following discrete analogue of Theorem 2 leads to a criterion for S-determinateness which complements Theorem 4. Denote the atoms of $F$ by $a(j)(j=$ $0,1, \ldots)$ and suppose they are ultimately ordered, that is, that there exists $j^{\prime}$ such that $a(j)<a(j+1)$ if $j \geq j^{\prime}$. We do not assume the atoms are $h$-dense, but rather that $a=\lim _{j \rightarrow \infty} a(j) / a(j-1) \geq 1$. The atoms comprise a lacunary sequence if $a>1$.

Discrete rapidly varying laws are defined by

(1D) $p_{j}=A_{j} \exp \left[-\eta_{j}-\sum_{i=j^{\prime}}^{j} \zeta_{i} / i\right] \quad\left(j \geq j^{\prime} \geq 0\right)$,

where $A_{j} \rightarrow A \in(0, \infty),\left\{\eta_{j}\right\}$ is a positive and non-decreasing sequence, and $0<\zeta_{j} \rightarrow \infty$.

Let $\rho_{j}=\log (a(j) / a(j-1))$ and assume further that

(2D) $\mu_{n}=\sum_{j}(a(j))^{n} p_{j}<\infty \quad(n \in \mathbb{N})$.

(3D) For a constant $c>0, \sum_{j \geq j} \eta_{j} /(a(j))^{c}<\infty$.

(4D) There exists a constant $m \geq 1$ such that $\sum_{j \geq y^{\prime}}(a(j))^{-m}<\infty$.

(5D) There exist $N \in \mathbb{N}$ and integers $x_{n}(n \geq N)$ such that $x_{n} \uparrow \infty$ and

$$
n \leq \zeta_{j} / j \rho_{j} \leq n+1 \text { if and only if } x_{n} \leq j \leq x_{n+1} .
$$

These conditions are essentially the lightest for which the following proof will work. We comment below on circumstances under which (3D)-(5D) are satisfied.

THEOREM 6. Suppose conditions (1D)-(5D) are satisfied. Then $\sigma(\mathscr{M} ; c)=\infty$ if

$$
\sigma(F ; c)=\sum_{j \geq j^{\prime}}(a(j))^{-c} j^{-1} \zeta_{j}=\infty .
$$

PROOF. As in the proof of Theorem 2, let $\bar{p}_{j}=A p_{j} / A_{j}$ and define $\phi_{n}(j)=$ $(a(j))^{n} \bar{p}_{j}$. Then

$$
-\log \left[\phi_{n}(j) / \phi_{n}(j-1)\right]=\eta_{j}-\eta_{j-1}+\zeta_{j} / j-n \rho_{j} .
$$

It follows from (5D) that $\phi_{n}(j)$ is non-increasing for $j \geq x_{n}(n \geq N)$. Using (4D) it follows that

$$
\begin{aligned}
\mu_{n} & \leq 2\left[\left(a\left(x_{n}\right)\right)^{n}+\phi_{n+m}\left(x_{n}\right) \sum_{j>x_{n}}(a(j))^{-m}\right] \\
& =2\left(a\left(x_{n}\right)\right)^{n}\left[1+\left(a\left(x_{n}\right)\right)^{m} p_{x_{n}} \sum_{j>x_{n}}(a(j))^{-m}\right] \leq 2\left(a\left(x_{n}\right)\right)^{n}(1+o(1)) .
\end{aligned}
$$

The assertion follows if $\sum\left(a\left(x_{n}\right)\right)^{-c}=\infty$. But

$$
\sigma(F ; c)=\sum_{n>N} \sum_{j=1+x_{n-1}}^{x_{n}}(a(j))^{-c} j^{-1} \zeta_{j} \leq \sum_{n>N} n \sum_{j=1+x_{n-1}}^{x_{n}}(a(j))^{-c} \rho_{j} .
$$


Observing that for $a<b$,

we have that

$$
a^{-c} \log (b / a)=a^{-c} \int_{a}^{b} x^{-1} d x \leq \int_{a}^{b} x^{-1-c} d x=\left(a^{-c}-b^{-c}\right) / c,
$$

$$
\begin{aligned}
\sigma(F ; c) & \leq c^{-1} \sum_{n>N} \sum_{j=1+x_{n-1}}^{x_{n}}\left[(a(j-1))^{-c}-(a(j))^{-c}\right] \\
& =c^{-1} \sum_{n>N} n\left[\left(a\left(x_{n-1}\right)\right)^{-c}-\left(a\left(x_{n}\right)\right)^{-c}\right]=c^{-1} \sum_{n>N}\left(a\left(x_{n}\right)\right)^{-c}+O(1) .
\end{aligned}
$$

Condition (3D) is imposed to ensure that the infinitude of $\sigma(\mathscr{M} ; c)$ is controlled by the index sequence $\left\{\zeta_{j}\right\}$. Condition (4D) is satisfied with $m=1$ when $a>1$ and with possibly larger values of $m$ when

$$
a(j)=j^{\Delta} l(j),
$$

$\Delta>0$, and $l(\cdot)$ is slowly varying at infinity. Lattice laws correspond to the case $\Delta=1$ and $l(\cdot)$ a constant, the lattice spacing. If (9) holds, then $j \rho_{j}=\Delta+o(1)$ $(j \rightarrow \infty)$, and hence Condition (5D) holds provided $\zeta_{j}$ is eventually increasing to $\infty$. Condition (5D) is also satisfied if $1<a<\infty$ and $\zeta_{j} / j$ is eventually increasing to $\infty$.

Theorem 6 implies the following sufficient condition that a discrete distribution $F$ be S-det.

COROLLARY 3. If $F$ satisfies Conditions (1D)-(5D) with $c=1 / 2$, then it is SC-det provided $\sigma(F ; 1 / 2)=\infty$.

EXAMPLE 4. Suppose $A_{j} \equiv A, \eta_{j} \equiv 0, \zeta_{j}=j \beta^{j}$ and $a(j)=\alpha^{j}$, where $\alpha, \beta>1$. Then $F$ is $\mathrm{S}$-det provided $\beta \geq \sqrt{\alpha}$. It seems likely that $F$ is $\mathrm{S}$-indet when the inequality is reversed. However a Krein criterion is not applicable because the atoms do not satisfy the $h$-density condition.

EXAMPLE 5. This is the same as Example 4 but now with $a(j)=j$ and $\zeta_{j}=j^{1 / \lambda}$, $\lambda>0$. Then $-\log p_{j} \sim \lambda j^{1 / \lambda}$ and we see that (8) is satisfied if $\lambda>2$, that is, $F$ is S-indet. Conversely $\sigma(F ; 1 / 2)=\infty$ if $\lambda \leq 2$, so $F$ is S-det. If instead $a(j)=j^{2}$ then $\sum\left(-\log p_{j}\right) /(1+a(j))<\infty$ if and only if $\lambda>1$, and $\sigma(F ; 1 / 2)=\infty$ if and only if $\lambda \leq 1$.

\section{The Hamburger problem}

In this section we assume that $F$ is a two-sided DF. Before discussing applications of Theorem 1 and Theorem 2 to the Hamburger problem we summarize some known 
work relevant to our theme. Sjödin [14] shows that $H C(\mathscr{M})$ is 'sharp in a certain sense'. Let $\beta_{2 n}=\sup _{j \geq n} \mu_{2 j}^{-1 / 2 j}$. Another criterion of Carleman is that $F$ is H-det if $\sum_{n} \beta_{2 n}=\infty$. Sjödin [14] shows there is a non-determining moment sequence $\left\{\lambda_{n}\right\}$ satisfying $\lim _{n \rightarrow \infty}\left(\lambda_{2 n} / \mu_{2 n}\right)^{1 / n}=0$ if and only if $\sum \beta_{2 n}<\infty$. This assertion implies that $\sum \mu_{2 n}^{-1 / 2 n}<\infty$, but since Carleman's condition is not necessary, it can be that $\left\{\mu_{n}\right\}$ is determining.

An integral condition similar in form to the Krein criterion, and due to M. Riesz (Koosis [7, pages 132-142]) is necessary and sufficient for $\mathscr{M}$ to be determining. Let $\mathscr{P}$ be the set of all polynomials $P(x)=\sum_{j=0}^{N} c_{j} x^{j}(N=1,2, \ldots)$ where the coefficients are arbitrary complex numbers. Then

Define Riesz' function

$$
\int_{\mathbb{R}}|P(x)|^{2} d F(x)=\sum_{i, j=0}^{N} \mu_{i+j} c_{i} \bar{c}_{j} .
$$

$$
\mathscr{R}(x)=\sup _{P \in \mathscr{P}}\left\{|P(x)|^{2}: \int_{\mathbb{R}}|P(x)|^{2} d F(x) \leq 1\right\} .
$$

Riesz' theorem asserts that $F$ is $\mathrm{H}$-det if and only if

$$
\int_{\mathbb{R}} \frac{\log ^{+} \mathscr{R}(x)}{1+x^{2}} d x=\infty .
$$

Of course obtaining the functional form of $\mathscr{R}(\cdot)$ in any particular case is virtually impossible. Koosis shows that both the Carleman and Krein criteria follow from this result.

It is worth recalling that the classical Krein condition (1) makes a very strong analytical assertion about the functional form of the density function $f(\cdot)$ since (1) holds if and only if $f(x)=|h(x)|$ a.e. where the function $h(z)$, iz $>0$, belongs to the Hardy space $H^{1}$ (Garnett [4, page 66]). Lin [8] uses this fact in his proof of the Krein criterion, together with the fact that $\int_{\mathbb{R}} h(x) e^{i \theta x} d x=0$ for all $\theta \geq 0$.

Gabardo [3, Theorem 2.4] proved a converse version of the Krein criterion by showing that if a moment sequence $\mathscr{M}$ is not $\mathrm{H}$-determining then there are distinct density functions $w^{\lambda}(x)$, indexed by all complex $\lambda$ with $\Im \lambda>0$, which have this moment sequence and $I\left(w^{\lambda} ; 0\right)<\infty$.

Corollary 1 with $c=1 / 2$ and $l=2$ yields a small extension of Koosis' [7] converse Carleman criterion for laws which have a symmetric density function, more precisely, if $f(-x)=f(x)$ when $x>x^{\prime}$. The symmetry assumption can be relaxed provided certain balance conditions are imposed on the extreme tails of $F$. See Pakes et al. [11] for examples of the classification of some non-symmetric distributions.

We assume

$$
\sigma_{H}(\mathscr{M})=\sum_{n \geq 0} \mu_{2 n}^{-1 / 2 n}<\infty
$$


and our task is to find additional conditions which ensure that $H K(f)$ holds. The first such result does this through a condition on the density function.

THEOREM 7. Suppose there exists $y^{\prime}>-\infty$ such that (H1) $-\log \left[f\left(e^{y}\right)+f\left(-e^{y}\right)\right]$ is convex in $\left[y^{\prime}, \infty\right)$; and (H2) there exist constants $x^{\prime}>0,0<A, B<1$ and $0<c_{1}<c_{2}<\infty$ such that

$$
c_{1} \exp \left(-x^{A}\right) \leq f(-x) / f(x) \leq c_{2} \exp \left(x^{B}\right) \quad\left(x \geq x^{\prime}\right) .
$$

Then $F$ is $H K$-indet if $\sigma_{H}(\mathscr{M})<\infty$.

PROOF. Let $X$ be a random variable whose density function is $f(\cdot)$ Then $Y=X^{2}$ has the moment sequence $\left\{\mu_{2 n}\right\}$ and its DF $H(\cdot)$ has the density function

$$
h(x)=\frac{1}{2 \sqrt{x}}[f(\sqrt{x})+f(-\sqrt{x})] \quad(x>0) .
$$

Condition (H1) ensures that $h(\cdot)$ satisfies the conditions of Theorem 3, and $H$ is SK-indet. But $S K(h)$ can be expressed as

$$
\int_{x^{\prime}}^{\infty} x^{-2}[-\log (f(x)+f(-x))] d x<\infty .
$$

However, (H2) implies that for $x>x^{\prime}$,

$$
-\log f(x)<\log \left(1+c_{2} \exp \left(x^{B}\right)\right)-\log (f(x)+f(-x))
$$

and hence $\int_{x^{\prime}}^{\infty} x^{-2}(-\log f(x)) d x<\infty$. Finitude of the integral over $\left(-\infty,-x^{\prime}\right]$ follows in a similar manner, and hence $H K(f)$ is satisfied.

The last part of the proof establishes that under the balance condition $(\mathrm{H} 2)$, the distribution of the two-sided random variable $X$ is HC-det if and only if the distribution of $X^{2}$ is SC-det. This strengthens Theorem 3 in Pakes et al. [11].

EXAMPLE 6. Denote the generalized gamma density function by

$$
g(x ; a, \lambda)= \begin{cases}k_{a}^{-1} x^{a-1} \exp \left(-x^{1 / \lambda}\right) & \text { if } x>0 \\ 0 & \text { if } x<0,\end{cases}
$$

where $a, \lambda>0$ and $k_{a}=\Gamma(a \lambda)$. Its $n$-th order moment is $v(n ; a, \lambda)=\Gamma(\lambda(n+$ $a)) / \Gamma(a \lambda)$. Now let $0<p=1-q<1$ and define the two-sided density

$$
f(x)= \begin{cases}p g(x ; a, \lambda) & \text { if } x>0 \\ q g(-x ; b, \lambda) & \text { if } x<0\end{cases}
$$


where $b>0$. Conditions (H1) and (H2) are both satisfied. Supposing $a>b$, we find from

$$
\mu_{2 n}=p v(2 n ; a, \lambda)+q v(2 n ; b, \lambda)
$$

that $\mu_{2 n}^{-1 / 2 n} \sim$ const $\cdot n^{-\lambda}$, and hence $F$ is HK-indet if $\lambda>1$. This is an alternative to the treatment outlined by Pakes et al. [11].

EXAMPLE 7. Example 6 can be extended by defining

$$
f(x)= \begin{cases}p g\left(x ; a, \lambda_{+}\right) & \text {if } x>0 \\ q g\left(-x ; b, \lambda_{-}\right) & \text {if } x<0,\end{cases}
$$

where $\lambda_{+} \neq \lambda_{-}$. The convexity condition is still satisfied for all positive $\lambda_{ \pm}$. However the balance condition requires that $\lambda_{ \pm}>1$ and then $\sigma_{H}(\mathscr{M})<\infty$, showing that $F$ is $\mathrm{HK}$-indet under this condition. However it is not clear that the converse Carleman condition can handle other combinations of $\lambda_{ \pm}$. For example, Pakes et al. [11] observe that $F$ is HK-indet if $\lambda_{+}>2$ or $\lambda_{-}>2$. The Carleman condition is satisfied if $\lambda_{ \pm} \leq 1$, leaving the cases $\lambda_{+} \leq 1 \leq \lambda_{-} \leq 2$ and $\lambda_{-} \leq 1 \leq \lambda_{+} \leq 2$ undecided.

Our second approach assumes information is available to compare the partial moments

$$
\mu_{n}^{+}=\int_{0}^{\infty} x^{n} f(x) d x \quad \text { and } \quad \mu_{n}^{-}=\mu_{n}-\mu_{n}^{+} .
$$

THEOREM 8. Suppose $f(x)$ and $f(-x)(x \geq 0)$ each satisfy the convexity condition (b) of Theorem 1, and that there are constants $0<b<1<B<\infty$ and $n^{\prime} \in \mathbb{N}$ such that

(H3) $b^{2 n} \mu_{2 n}^{+} \leq \mu_{2 n}^{-} \leq B^{2 n} \mu_{2 n}^{+}$.

Then $F$ is $H K$-indet if $\sigma_{H}(\mathscr{M})<\infty$.

ProOF. The assumptions imply that

$$
\sum_{n \geq n^{\prime}}\left(\mu_{2 n}^{ \pm}\right)^{-1 / 2 n}<\infty
$$

thus fulfilling the conditions of Corollary 2 for each of $f(x)$ and $f(-x)$ and hence satisfying $H K(f)$.

Example 6 satisfies the conditions of Theorem 8 since $\mu_{2 n}^{+} / \mu_{2 n}^{-} \sim$ const $\cdot n^{\lambda(a-b)}$. On the other hand, Example 7 does not satisfy (H3) because

$$
\mu_{2 n}^{+} / \mu_{2 n}^{-} \sim \text { const } \cdot n^{a \lambda_{+}-b \lambda_{-}}(n / e)^{\left(\lambda_{+}-\dot{\lambda}_{-}\right) n} .
$$


Taking the $1 / 2 n$ root leaves a factor $n^{\lambda_{+}-\lambda_{-}}$which could upset the convergence of one or other of the series $\sum_{n \geq n^{\prime}}\left(\mu_{2 n}^{ \pm}\right)^{-1 / 2 n}$. More generally, if $\sum_{n \geq n^{\prime}}\left(\mu_{2 n}^{+}\right)^{-1 / 2 n}<\infty$ say, then $\sum_{n \geq n^{\prime}} \mu_{2 n}^{-1 / 2 n}$ will converge even if $\sum_{n \geq n^{\prime}}\left(\mu_{2 n}^{-}\right)^{-1 / 2 n}=\infty$. Of course, convergence of both partial moment series could possibly be established from other information.

The next result states two versions of the converse Krein criterion for the Hamburger problem. Its proof permutes steps in the two previous proofs and hence it is omitted. Just note that each of the conditions (I) and (II) below is a substitute for (H1).

THEOREM 9. Let $f(\cdot)$ be a two-sided density function with finite moments of all orders and suppose that for some $x^{\prime}>0$,

$$
\int_{x^{\prime}}^{\infty} x^{-2}(-\log f(x)) d x=\infty \quad \text { or } \quad \int_{x^{\prime}}^{\infty} x^{-2}(-\log f(-x)) d x=\infty .
$$

Suppose also that either:

(I) (H3) holds, and (see (9)) $h(x) \in K R_{-\infty}$ satisfies the conditions of Theorem 2; or that

(II) (H2) holds and either $f(x)$ or $f(-x)\left(x \geq x^{\prime}\right)$ are in $K R_{-\infty}$ and satisfy the conditions of Theorem 2. Then $F$ is HC-det.

\section{Logarithmic and exponential transformations}

Logarithmic transformations are often used to stabilize the variance of data sets. Exponentiation is used to obtain one-sided distributions with long tails, such as the log-normal and log-gamma laws. In this section we round off our account with a quick look at how these transformations affect moment determinateness.

Let $Y>0$ be a random variable with DF $H$ and a density function $h(y)$ positive for all sufficiently large $y$. This is related to the density function $f(x)$ of $X=\log Y$ through the relation $h(y)=y^{-1} f(\log y)$. Applying the Krein criterion to $f(x)$ shows that $F$ is $\mathrm{HK}$-indet if there are constants $0<d, \delta<1$ and slowly varying functions $L(x)$ and $M(x)$ such that

$$
h(y) \geq \begin{cases}y^{-1} \beta(y, \delta, L)=y^{-1} \exp \left[-(\log y)^{1-\delta} L(\log y)\right] & \text { if } y>y^{\prime}=e^{x^{\prime}} \\ y^{-1} \beta\left(y^{-1}, d, M\right) & \text { if } 0<y<y^{\prime \prime}=e^{-x^{\prime}} .\end{cases}
$$

Thus $F$ can be $\mathbf{H}$-indet only if $H$ allocates large mass in the region of zero and $+\infty$. Conversely, $I_{H}\left(f ; x^{\prime}\right)=\infty$ if

$$
h(y) \leq \begin{cases}y^{-1} \beta(y, 1, L) & \text { if } y>y^{\prime} \\ y^{-1} \beta\left(y^{-1}, 1, M\right) & \text { if } 0<y<y^{\prime \prime}\end{cases}
$$


and either $\int_{x^{\prime}}^{\infty} L(x) d x / x=\infty$ or $\int_{x^{\prime}}^{\infty} M(x) d x / x=\infty$. Satisfaction of the auxilliary conditions of Theorem 9 is problematic. However if $M(x) \equiv L(x)\left(x>x^{\prime}\right)$ and $L(\cdot)$ is a normalized slowly varying function, then $F$ is HC-det.

Now let $Y$ take values in $\mathbb{R}$ and $X=e^{Y}$, giving $f(x)=x^{-1} h(\log x)$. Also, $\mu_{n}=\phi(n)$ where $\phi(\theta)=E(\exp (\theta Y))$ is the moment generating function of $Y$, assumed finite if $\theta>0$. Then $F$ is SC-det if $\sum(\phi(n))^{-1 / 2 n}=\infty$, and it is SK-indet if this sum is finite and $\psi(y)=y-\log h(y)$ is convex in $\left(y^{\prime}, \infty\right)$. Alternatively, $F$ is SK-indet if $\int_{y^{\prime}}^{\infty} e^{-3 y / 2}(-\log h(y)) d y<\infty$.

Suppose

$$
h(y)=e^{y} \hat{A}(y) \exp \left[-\hat{\eta}(y)-\int_{y^{\prime}}^{y} \xi\left(e^{v}\right) d v\right] \quad\left(y>y^{\prime}\right),
$$

where $\hat{A}(y) \rightarrow A \in(0, \infty), \hat{\eta}(\cdot)$ is non-decreasing and $\int_{y^{\prime}}^{\infty} e^{-3 y / 2} \hat{\eta}(y) d y<\infty$, and $\xi(x)>0$ and $(\log x) \xi(x) \uparrow \infty$. Then $F$ is S-det if and only if $\int_{x^{\prime}}^{\infty} x^{-5 / 2} \xi(x) d x$.

We end with an example which imbeds the indeterminate log-normal distribution as a boundary point of a larger family of distributions. Let $S(\alpha)$ denote a random variable having the extreme, or spectrally positive, stable law with index $\alpha \in(0,2]$. We use the normalization giving the Laplace transform

$$
E\left(e^{-t S(\alpha)}\right)= \begin{cases}\exp \left(t^{\alpha} \operatorname{sgn}(\alpha-1)\right) & \text { if } \alpha \neq 1 \\ \exp (t \log t) & \text { if } \alpha=1\end{cases}
$$

These expressions can be obtained from the form of the characteristic function of $S(\alpha)$ as given by Zolotarev [19, page 75], for example.

Now let $X=e^{-S(\alpha)}$, whose distribution may be called a log-stable law. More precisely it is a log-(spectrally-positive stable) distribution since the Lévy measure of $S(\alpha)$ is carried on $\mathbb{R}_{+}$. These distributions arise as interesting cases in a characterization problem involving a generalized form of length biasing (Pakes [10]). According to Samorodnitsky and Taqqu [13, page 52], they are used in modelling multifractals. A question of interest is for which values of $r>0$, if any, is the distribution $F_{r}$ of $X^{r}$ S-det? The parameter $r$ is effectively a scale constant for the distribution $H_{\alpha}$ of $S(\alpha)$. No extra generality is gained by including a location parameter.

The case $\alpha=2$ gives the log-normal distribution, and then $F_{r}$ is SK-indet for all $r$. If $\alpha<1$ then $S(\alpha)$ is positive, whence $0<X^{r}<1$ and $F_{r}$ is SC-det for all $r$. The outcome for the cases $1 \leq \alpha<2$ is described by the following result.

THEOREM 10. Suppose $X$ has the log-stable law described above. If $1<\alpha<2$ then $F_{r}$ is $S K$-indet for all $r>0$. If $\alpha=1$ then $F_{r}$ is $S C$-det if $r \leq 2$ and it is $S K$-indet if $r>2$. 
PROOF. The proof follows similar lines to the case $\alpha=1$ discussed by Pakes [10, Lemma 5.1 and Lemma 5.2]. The density function of $X^{r}$ is

$$
f_{r}(x)=(r x)^{-1} h\left(-\log x^{1 / r} ; \alpha\right) \quad(x>0),
$$

where $h(y ; \alpha)$ is the density function of $S(\alpha)$. This shows that we need only consider the form of $h(y ; \alpha)$ for $y<0$. In this case we have when $1<\alpha<2$ the integral representation (Zolotarev [19, page 74]): For $y>0$,

$$
h(-y ; \alpha)=\frac{\alpha y^{1 /(\alpha-1)}}{2(\alpha-1)} \int_{-\theta}^{1} U(p) \exp \left[-U(p) y^{\alpha /(\alpha-1)}\right] d p,
$$

where $\theta=(2-\alpha) / \alpha$ and

$$
U(p)=\left[\frac{2 \cos (\pi p / 2)}{\sin (\pi \alpha(p+\theta) / 2)}\right]^{\alpha /(\alpha-1)} \cdot \frac{\cos [\pi((\alpha-1) p+2-\alpha) / 2]}{\cos (\pi p / 2)} .
$$

This function decreases from infinity at $p=-\theta$ to zero at $p=1$. To see this use logarithmic differentiation of $U(p)$ with respect to $p$ to see that the sign of its derivative is the same as that of

$$
\xi(p)=\alpha^{2} \cot (\pi \alpha(p+\theta) / 2)+(\alpha-1)^{2} \tan (\pi((\alpha-1) p+2-\alpha) / 2)+\tan (\pi p / 2) .
$$

Rewrite this in terms of $x=\pi \alpha(1-p) / 2$ and use the symmetry relations $\cot (\pi-x)=$ $-\cot x$ and $\tan (\pi / 2-x)=\cot x$ to obtain

$$
\xi(p)=-\alpha^{2} \cot x+(\alpha-1)^{2} \cot \left(\left(1-\alpha^{-1}\right) x\right)+\cot (x / \alpha)=\frac{(\alpha D-C)^{2}+(\alpha-1)^{2}}{C-D},
$$

where $C=\cot (x / a), D=\cot x$ and we have used the addition formula $\cot ((1-$ $\left.\left.\alpha^{-1}\right) x\right)=(C D+1) /(C-D)$. But $0<x<\pi$ if $-\theta<p<1$, and hence $C>D$ since $\cot x$ decreases in $(0, \pi)$ and $x / \alpha<x$.

The substitution $z=U(p)$ gives the differential relation $d p=(\alpha / 2) A_{-}(d z)$ where $A_{-}(z)$ is a distribution function with support $\mathbb{R}_{+}$. We thus obtain the mixture representation

$$
h(-y ; \alpha)=\frac{y^{1 /(\alpha-1)}}{\alpha-1} \int_{0}^{\infty} z \exp \left(-z y^{\alpha /(\alpha-1)}\right) A_{-}(d z) .
$$

[There is a similar representation for $h(y ; \alpha)$ when $y>0$ but with a different mixing DF.] This representation yields

$$
f_{r}(x)=\alpha^{-1} \int_{0}^{\infty} f(x \mid z) A_{-}(d z) \quad(x>1)
$$


where

$$
f(x \mid z)=\frac{\alpha}{\alpha-1} \cdot \frac{z}{x}(\log x)^{1 /(\alpha-1)} \exp \left(-z(\log x)^{\alpha /(\alpha-1)}\right) .
$$

This clearly satisfies $S K(f(\cdot \mid z))$ for all $z>0$, and hence the mixed DF $F_{r}$ is SK-indet. See Pakes et al. [11] for a discussion of the decidability of mixtures.

Observe that the moment sequence $\mathscr{M}_{r}$ of $X^{r}$ is given by

$$
\mu_{n}(r)= \begin{cases}\exp \left[(r n)^{\alpha}\right] & \text { if } 1<\alpha<2 \\ (r n)^{r n} & \text { if } \alpha=1\end{cases}
$$

whence $\sigma_{S}\left(\mathscr{M}_{r}\right)<\infty$ if $1<\alpha<2$ or if $\alpha=1$ and $r>2$. The convexity condition required for Theorem 3 is that $-\log h(-y ; \alpha)$ is convex in $\left(y^{\prime}, \infty\right)$ for some $y^{\prime}>-\infty$. But the analysis required to prove this certainly exceeds that needed for a direct application of the Krein criterion. This illustrates the practical difficulties of using the converse criteria developed in previous sections-usually the direct criteria supplemented by tail comparisons suffice to resolve the situation for a given family of distributions. However we have noted cases of two-sided laws with unbalanced extreme tails where neither direct or converse criteria have been successful.

We close by remarking that there is an obvious Hamburger version of Theorem 10 where $r=1$ is the critical value separating determinateness and indeterminateness when $\alpha=1$.

\section{Acknowledgement}

I thank Dr Gwo Dong Lin for detecting a couple of errors in an earlier 'proof' of Theorem 1, and Dr Henrik Pedersen for remarks leading to a much improved version of Proposition 2.

\section{References}

[1] C. Berg, 'Indeterminate moment problems and the theory of entire functions', J. Comput. Appl. Math. 65 (1995), 27-55.

[2] N. H. Bingham, C. M. Goldie and J. L. Teugels, Regular variation (Cambridge Univ. Press, Cambridge, 1987).

[3] J.-P. Gabardo, 'A maximum entropy approach to the classical moment problem', J. Funct. Anal. 106 (1992), 80-94.

[4] J. B. Garnett, Bounded analytic functions (Academic Press, New York, 1981).

[5] C. C. Heyde. 'Some remarks on the moment problem, (I) and (II)', Quart. J. Math. Oxford (2), 14 (1963). 91-105. 
[6] J.-B. Hiriart-Urruty and C. Lemaréchal, Convex analysis and minimization algorithms I (Springer, Berlin, 1993).

[7] P. Koosis, The logarithmic integral I (Cambridge Univ. Press, Cambridge, 1988).

[8] G. D. Lin, 'On the moment problems', Statist. Probab. Letters 35 (1997), 85-90.

[9] A. G. Pakes, 'Length biasing and laws equivalent to the log-normal', J. Math. Anal. Appl. 197 (1996), 825-854.

[10] _ _ 'Characterization by invariance under length-biasing and random scaling', J. Statist. Plann. Inference 63 (1997), 285-310.

[11] A. G. Pakes, W.-L. Hung and J.-W. Wu, 'Criteria for the unique determination of probability distributions by moments', Aust. N. Z. J. Stat. 43 (2001), 101-111.

[12] H. L. Pedersen, 'On Krein's theorem for indeterminacy of the classical moment problem', $J$. Approx. Theory 95 (1998), 90-100.

[13] G. Samorodnitsky and M. S. Taqqu, Stable non-Gaussian random processes (Chapman and Hall, New York, 1994).

[14] T. Sjödin, 'A note on the Carleman condition for determinacy of moment problems', Ark. Mat. 25 (1987), 289-294.

[15] E. V. Slud, 'The moment problem for polynomial forms in normal random variables', Ann. Probab. 21 (1993), 2200-2214.

[16] M. D. Springer, The algebra of random variables (Wiley, New York, 1979).

[17] J. Stoyanov, Counterexamples in probability, 2nd Edition (Wiley, Chichester, 2000).

[18] —_ 'Krein condition in probabilistic moment problems', Bernoulli 6 (2000), 939-949.

[19] V. M. Zolotarev, One-dimensional stable distributions (American Math. Society, Providence, RI, 1986).

Department of Mathematics and Statistics

University of Western Australia

35 Stirling Highway, WA 6009

Australia

e-mail: pakes@maths.uwa.edu.au 